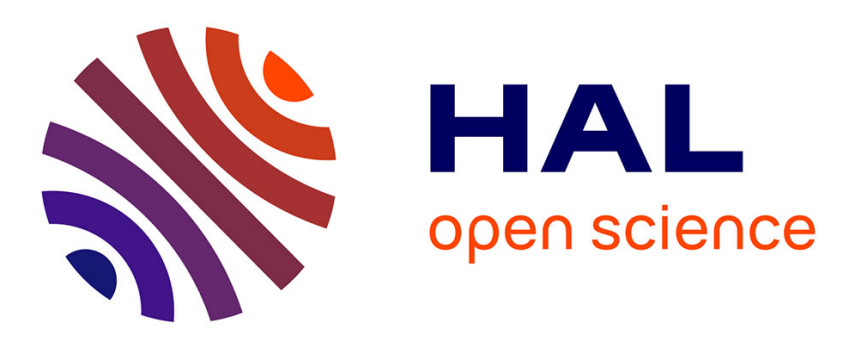

\title{
Transient Thermoelectric Generator: An Active Load Story
}

John Stockholm, Christophe Goupil, Pascal Maussion, Henri Ouerdane

\section{To cite this version:}

John Stockholm, Christophe Goupil, Pascal Maussion, Henri Ouerdane. Transient Thermoelectric Generator: An Active Load Story. Journal of Electronic Materials, 2015, vol. 44 ( $\left.{ }^{\circ} 6\right)$, pp. 17681772. 10.1007/s11664-014-3545-z . hal-01308074

\section{HAL Id: hal-01308074 https://hal.science/hal-01308074}

Submitted on 27 Apr 2016

HAL is a multi-disciplinary open access archive for the deposit and dissemination of scientific research documents, whether they are published or not. The documents may come from teaching and research institutions in France or abroad, or from public or private research centers.
L'archive ouverte pluridisciplinaire HAL, est destinée au dépôt et à la diffusion de documents scientifiques de niveau recherche, publiés ou non, émanant des établissements d'enseignement et de recherche français ou étrangers, des laboratoires publics ou privés. 


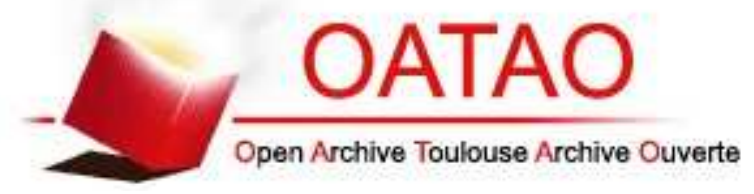

\section{Open Archive TOULOUSE Archive Ouverte (OATAO)}

OATAO is an open access repository that collects the work of Toulouse researchers and makes it freely available over the web where possible.

This is an author-deposited version published in : http://oatao.univ-toulouse.fr/ Eprints ID : 14328

To link to this article : DOI: $10.1007 / \mathrm{s} 11664-014-3545-\mathrm{z}$

URL : http://dx.doi.org/10.1007/s11664-014-3545-z

To cite this version : Stockholm, John and Goupil, Christophe and

Maussion, Pascal and Ouerdane, Henri Transient Thermoelectric

Generator: An Active Load Story. (2015) Journal of Electronic

Materials, vol. 44 ( ${ }^{\circ}$ 6). pp. 1768-1772. ISSN 0361-5235

Any correspondance concerning this service should be sent to the repository administrator: staff-oatao@ listes-diff.inp-toulouse.fr 


\title{
Transient Thermoelectric Generator: An Active Load Story
}

\author{
J.G. STOCKHOLM, ${ }^{1,5}$ C. GOUPIL ${ }^{2}$ P. MAUSSION,${ }^{3}$ and H. OUERDANE ${ }^{2,4}$ \\ 1.-Marvel Thermoelectrics, 11 Rue Joachim du Bellay, Marsinval, 78540 Vernouillet, France. \\ 2.-Laboratoire Interdisciplinaire des Energies de Demain (LIED), UMR 8236 Université Paris \\ Diderot CNRS, 5 Rue Thomas Mann, 75013 Paris, France. 3.-Laboratoire Plasma et Conversion \\ d'Energie (LAPLACE), UMR 5213 INP Université de Toulouse CNRS, 2, rue Charles Camichel, \\ 31071 Toulouse, France. 4.- Russian Quantum Center, 100 Novaya Street, Skolkovo, \\ 143025 Moscow Region, Russia. 5.—e-mail: johngstockholm@gmail.com
}

Under stationary conditions, the optimization of maximum power output and efficiency of thermoelectric generators (TEG) is a well-known subject. Use of a finite-time thermodynamics (FTT) approach to the description of TEGs has demonstrated that there exists a closed feedback effect between the output electrical load value and the entering heat current. From the practical point of view, this effect is strongly evidenced by the use of direct current (DC-to-DC) converters as active loads. Both transient conditions and FTT contribute to a complex landscape of the optimization of the power and efficiencies of a TEG. It has been claimed that the use of inductive load may lead to a strong enhancement of the efficiency, and the frequency response of a TEG as a bandpass filter has also been recently reported. We consider these results using a classical linear Onsager approach of a TEG operating under transient conditions. We show that a trans-admittance may be defined as a coupling element between the input and the output, leading to the observed electric-to-thermal feedback. We discuss recent experiments on a TEG connected to an active load, which is reported to boast an efficiency exceeding the usual stationary DC thermoelectric efficiency.

Key words: Thermoelectric energy conversion, thermoelectric devices, thermodynamic constraints on energy production

\section{INTRODUCTION}

Claims have been made that an efficiency exceeding the direct current (DC) operation of thermoelectric generators (TEG) can be achieved, ${ }^{1}$ and a first theoretical approach explaining such a performance was proposed by Apostol and Nedelcu a few years ago. ${ }^{2}$ Thermoelectric generators are autonomous heat engines in the sense that their operation is not periodically driven as in classical cycles (e.g., Carnot), but rather corresponds to a nonequilibrium steady-state generated by externally imposed time-independent boundary conditions. ${ }^{3}$ The effects of the finite thermal conductance of the heat exchangers placed between the heat baths and the heat engines must be accounted for to obtain insight into the operation of TEGs under realistic working conditions. While the traditional Carnot-like approach, assuming fixed temperatures across the thermoelectric device, is clearly not appropriate since it imposes non-realistic thermal boundary conditions to the system, finite-time thermodynamics (FTT) permits full consideration of the presence of heat exchangers, ${ }^{4,5}$ and, in particular, a correct estimation of the distribution of the power inside a system.

From a fundamental point of view, the coupling between the thermal and electrical processes can be described as a closed feedback effect between the output electrical load value and the entering heat current. ${ }^{6}$ Using a combination of finite-time thermodynamics and Onsager force-flux framework, our approach leads to the conclusion that there exist optimal working conditions for thermoelectric generators, not only for the electrical but also for the 
coupled thermal conditions; this search for optimal working conditions reflects the power versus efficiency trade-off at the heart of FTT analyses: one does not seek maximum heat-to-work conversion efficiency, but optimum efficiency at maximum power output, since both are tightly tied together through their dependence on the working conditions. For thermoelectric generators in a standard setup, as depicted on Fig. 1, the optimization problem has been studied for the stationary mode $;^{6-9}$ TEGs thermally connected in series have also been studied. ${ }^{10}$

The output current is a central parameter for all stationary conditions, but it is also the case for a system working under transient conditions. The transient response has mainly been studied experimentally for Peltier heat pump devices and less for thermoelectric generators. The doctoral work of Paul Gray on the dynamic behaviour of thermoelectric devices was compiled and published in 1960 in a book in which a small-signal analysis for the thermoelectric generator and heat pumps is presented. ${ }^{11}$ This study and those that followed reveal that the frequency response presents a non-trivial shape, leading to the observation of a strong coupling between the output varying current and the performance of the system at low frequencies. Measurement techniques based on impedance spectroscopy and utilizing transmission line theory in electrical circuits for the characterization of thermoelectric materials and devices are particularly well suited as shown in Refs. 12-17. Jorge García Cañadas and Gao Min made a low frequency impedance spectroscopy analysis of thermoelectric modules, ${ }^{18}$ and Nedelcu and co-workers showed that the impedance response of a commercial (Hi-Z 20) thermoelectric module presents a very singular behaviour at high frequencies; ${ }^{19}$ see Fig. 2.

From these measurements Nedelcu found an equivalent series resistor, inductor, capacitor (RLC) electrical circuit, in which $R$ is the leakage part of the capacitance. The extracted values: $L=7.1 \mathrm{nH}$,
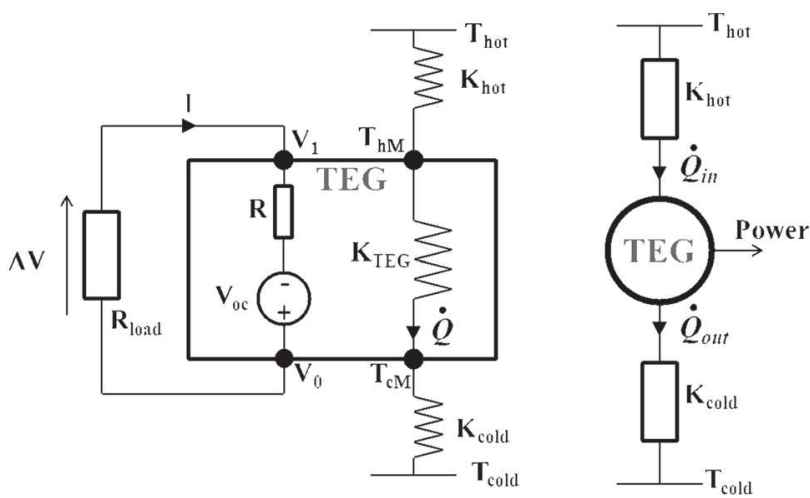

Fig. 1. Nodal representation of the thermoelectrical (left) and thermodynamical (right) pictures of the thermoelectric module and the load. Notice that the temperature difference at the TEG depends directly on the working point imposed by the output current.
$C=1.2 \mu \mathrm{F}$, and $R=0.21 \Omega$, are reasonable for an electrical network at the centimetre scale. It should be noticed that the central term is here the resistorinductor $(L C)$ resonance which defines a frequency range where the impedance is minimal. According to the electrical impedance matching rule, the use of an active complex load tuned in amplitude and phase may lead to specific performances. At least this may only be observed in thermoelectric systems with a sufficiently small internal resistance.

\section{MODEL}

To understand the physical mechanisms underlying the results and observations reported above, we analyse the response of the charge carrier distribution along a thermoelectric rod with a Seebeck coefficient $\alpha$ subjected to a temperature difference $\Delta T=T-T_{0}$, where $T_{0}$ is the cold side temperature. As an extensive parameter, this carrier distribution is directly linked to the quasi-Fermi levels (QFL) of the material (Fig. 3). Considering an applied output voltage, we have a resulting band curvature governed by the sum of the polarization $V_{p o l}$ and the Seebeck-induced voltage $V_{S}=\Delta V=\alpha \Delta T$. Under transient varying output voltage $V_{a p p}$, the carrier distribution along the rod is modified. The response of the carrier can be understood as a diffusion capacitance which we evaluate now.

Let us first examine the non-stationary Seebeck coefficient. For a small variation of the electrical conditions, we have $d \Delta V \equiv d V=d(\alpha \Delta T)=$ $d\left[\alpha\left(T-T_{0}\right)\right]$. Using the expression of the Thomson coefficient $\tau=d(\alpha T) / d T-\alpha$, we get:

$$
\left.d V=\left[\tau\left(1-T_{0} / T\right)-\alpha\right)\right] d T=\alpha_{S S} d T,
$$

where $\alpha_{S S}$ is the effective Seebeck coefficient for a small signal configuration, and we observe that it is a function of the Thomson contribution. To proceed, we consider that the charges within the thermoelectric rod form a non-interacting electrons gas, which may be characterized by thermoelastic coefficients defined in analogy with the classical gas

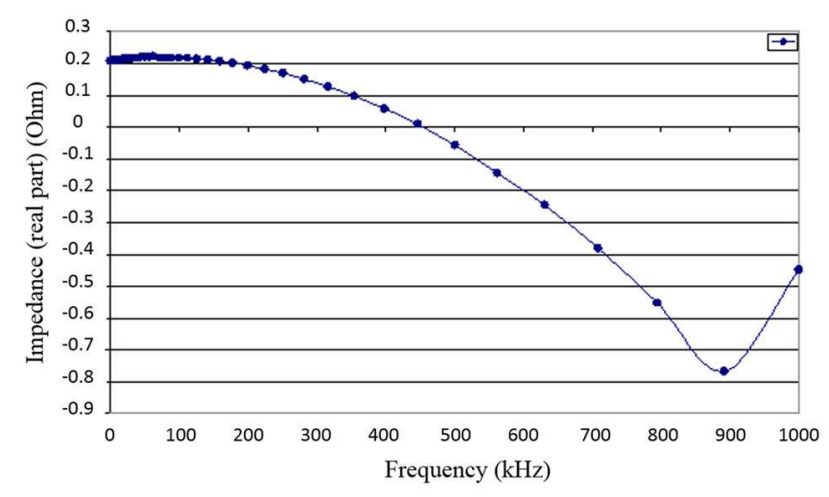

Fig. 2. Frequency-dependence of the modulus of the impedance for a module Hi-Z 20 (after Ref. 19). 
using the correspondence: electrochemical potential $\mu \equiv$ pressure and particle number $N \equiv$ volume. More precisely, we get: $\beta N=(\partial N / \partial T)_{\mu}$ : analogue to thermal dilatation coefficient (in $\mathrm{K}^{-1}$ ), and $\chi_{T} N=(\partial N / \partial \mu)_{T}$ : analogue to isothermal compressibility (in $\mathrm{J}^{-1}$ ), so that

$$
\alpha_{S S}=-\frac{1}{e}\left(\frac{\partial \mu}{\partial T}\right)_{N}=\frac{\beta}{e \chi_{T}},
$$

where $e$ is the electron charge. The next step is the description of the dynamics of population and temperature imbalance across the system under transient conditions, which satisfies:

$$
\tau_{0} \frac{\mathrm{d}}{\mathrm{d} t}\left(\begin{array}{c}
\Delta N_{e} \\
\Delta T
\end{array}\right)=-\left(\begin{array}{cc}
1 & -A \chi_{T} \\
-A \chi_{T} / \ell & \left(L_{\mathrm{o}}+A^{2}\right) / \ell
\end{array}\right)\left(\begin{array}{c}
\Delta N_{e} \\
\Delta T
\end{array}\right),
$$

where $\Delta N_{e}=e \Delta N$ and $A=\beta / \chi_{T}-e \alpha=e \tau\left(1-T_{0} / T\right)$ represents the effective thermoelectric coupling and the other parameters are given by: $\tau_{0}=e^{2} \chi_{T} / G$, $\ell=C_{N} / e^{2} \chi_{T} T$, and $L_{o}=K / G T$, with $K$ and $G$, respectively, being the thermal and electrical conductances of the thermoelectric element. The parameter $L_{o}$ is the Lorenz number associated to the transport properties, while $\ell$ is a quantity that derives from the thermoelastic properties of the electron gas and with the same dimension as $L_{o}$; $C_{N} N=T(\partial S / \partial T)_{N}$ is the analogue of the specific heat at constant volume. Note that $\tau_{0}$ gives an $R C$-like characteristic time of the system.

The solution of Eq. 3 yields the resulting electrical time constant, $\tau_{\text {elec }}=\lambda \tau_{0}$, of the system, with

$$
\lambda=\frac{C_{N} T}{\chi_{T} e^{2} \tau^{2}\left(T-T_{0}\right)^{2}} \approx \frac{3 N k_{B}^{2}}{e^{2} \tau^{2} \eta_{C}}
$$

in the strong coupling limit $A \gg L / \ell$, with $k_{B}$ being the Boltzmann constant and $\eta_{C}$ being the Carnot efficiency. Note that the approximate value is obtained in the non-degenerate semiconductor limit. Denoting $l$ the length of the thermoelectric elements, we obtain the following approximate expressions:

$$
\begin{gathered}
\tau_{\text {elec }} \approx \frac{3 n k_{B} l^{2}}{\sigma \tau^{2} \eta_{C}^{2} T} \\
C_{\text {diff }} \approx \frac{C_{N}}{\tau^{2} \eta_{C}^{2} T}
\end{gathered}
$$

for time constant of the system and the diffusion capacitance. We can now give an estimation of the diffusion capacitance $C_{\text {diff }}$. Assuming a nondegenerate semiconductor at room temperature with doping $n=5 \times 10^{19} \mathrm{~cm}^{-3}$ and effective mass $m_{0}=0.26 m_{e}$, where $m_{e}$ is the free electron mass, we get the expression of the heat capacitance of the electronic gas as $C_{N}=N k_{B} \frac{T}{T_{F}}$ where $T_{F} \approx 2208 \mathrm{~K}$ is the Fermi temperature estimated from the standard equation of state of the electrons. At room temperature, we obtain $C_{N}=616.10^{-7} \mathrm{JK}^{-1}$ and $\eta_{C}=1-\frac{400}{700}=0.43$. An estimation of the Thomson term is given by the approximation with the Seebeck coefficient by $S \approx \tau \eta_{C} \approx 150 \mu \mathrm{VK}^{-1}$. For a thermoelectric element of size $l=1 \mathrm{~cm}$, we finally obtain $C_{\text {diff }} \approx 7 \mathrm{~F}$. This very large value is still underestimated since we do not account for the lattice contribution in the calculation of the specific heat. Then, diffusion capacitances of order of hundreds of Farad are perfectly realistic, as previously reported. $^{19}$

\section{RESULTS AND DISCUSSION}

\section{Orders of Magnitude}

A numerical estimation of the characteristic associated frequency $f_{c}$ can be easily obtained. Assuming a material doping concentration of $5.0 \times 10^{17} \mathrm{~cm}^{-3}$ and a non-interacting electron gas, we get for a $1-\mathrm{cm}$ element, $f_{c}=6.3 \mathrm{kHz}$. This result merely gives an order of magnitude, and a more refined analysis should be undertaken. We also consider the measurements on a Hi-Z20 module (Fig. 4). The extracted parameters from the equivalent circuit depicted in Fig. 5 are $R=0.02, R_{T E}=0.0614$, $C_{T E}=1.91781 \mathrm{mF}$, and $L=0.165 \mu \mathrm{H}$. The characteristic frequency $f_{c}=1 / R C=6520 \mathrm{~Hz}$, is in excellent agreement with the prediction of the model. We can make a qualitative conclusion as regards the existence of a specific high-frequency response of a thermoelectric system, though more theoretical and experimental studies should be developed. Nevertheless, in contrast with the usual thermoelectric consideration of a unique internal resistance, the total internal impedance contains the $R_{T E}$ and $C_{T E}$ in parallel, as a low pass filtering. The self-inductance term just comes from the wiring of the thermoelectric system.

\section{DC-DC Converter}

We now consider the active load matching question from the DC-DC loading and optimization. Using a maximum power point tracking (MPPT) control, one can match the load impedance magnitude and phase in order to drive the maximum extraction of power from the TEG to flow down to the electrical load. ${ }^{18}$ The MPPT controller will automatically find the optimum voltage $V_{M P P}$ or current $I_{M P P}$ at which a TEG should operate to obtain the maximum power output under a given condition. A DC-DC converter is used to act as an interface between the load side and the TEG module. There are several control techniques such as, e.g., the perturb and observe (P\&O) and incremental conductance (INC). The conversion efficiencies are around $90 \%$. This work is extensively described in Ref. 20 .

\section{CASE OF A 4-KW GENERATOR}

A few years ago, a device boasting a high-efficiency conversion of heat flux to electrical power 


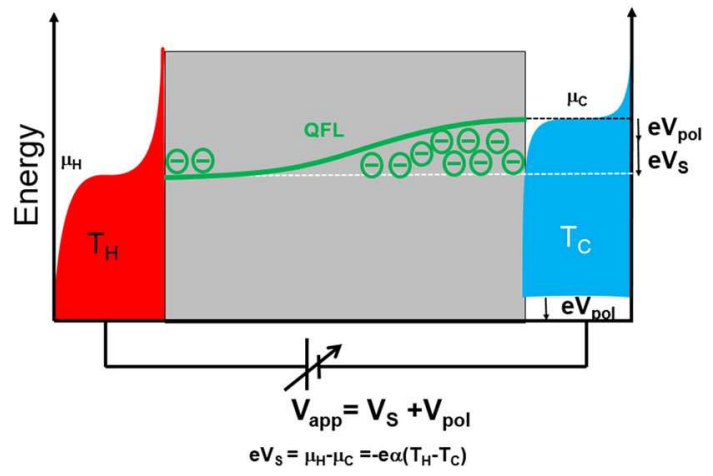

Fig. 3. Quasi-Fermi levels and applied voltage. The thermoelectric material is subjected to thermal and electrical biasing, leading to a redistribution of the free carriers.

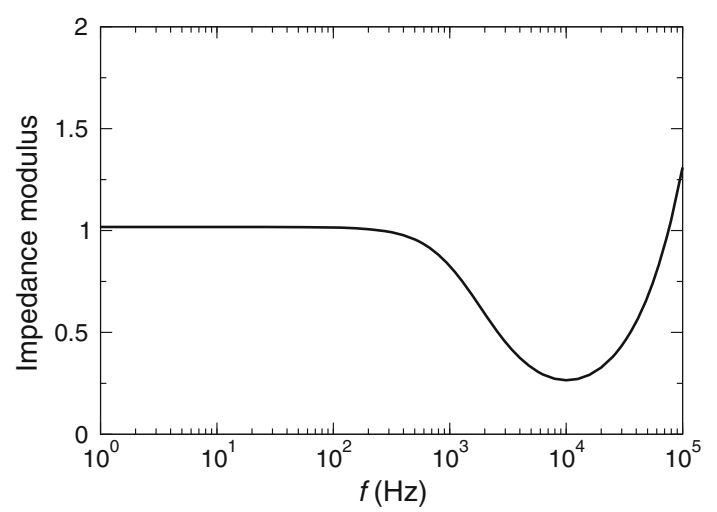

Fig. 4. Frequency-dependence of the modulus of the impedance normalized to its zero-frequency value.

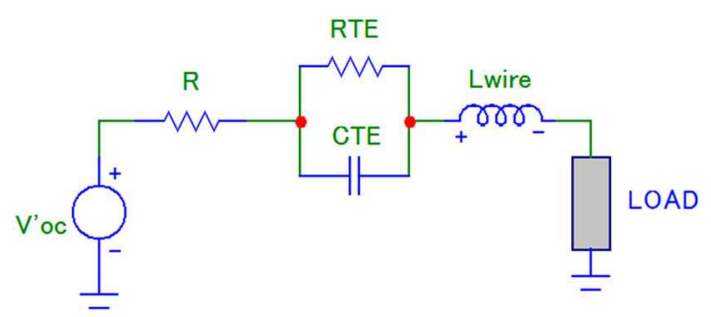

Fig. 5. Equivalent circuit derived from Fig. 4.

was proposed and patented. ${ }^{21}$ The claimed high performance is achieved with the use of a ring of metallic components and anodically sliced, reduced barriers, high purity $n$-type and $p$-type semiconductor wafers. The heat flux induced by heating one set of fins and cooling the other is extracted from a ring of bismuth telluride-based $n$-type wafers and antimony telluride-based $p$-type wafers using makebefore-break control of metal-oxide-semiconductor field-effect transistors (MOSFETs). This system combines both a very small internal resistance and an active load using switched MOSFET transistors. Attention should be paid to the output matching of the system based on a specific electrical transformer. This latter allows a thermoelectric conversion under very small voltage followed by a voltage elevation. A view of the system's geometry is shown in fig. 9 of the patent. ${ }^{21}$ Measurements were made in 2008 by Marin Nedelcu on the 4-kW system of Jon Schroeder, and these were recently repeated by John Stockholm on the same generator. ${ }^{1}$ The thermal power was measured by weighing a bottle of propane before and after the test which lasted about $1 \mathrm{~h}$. The $60-\mathrm{Hz}$ electrical power output from the $200-\mathrm{kHz}$ converter was measured on a resistive load of 2 banks in parallel of 20 filament lamps of $100 \mathrm{~W}$ each in parallel. The voltage was on each bank about $110 \mathrm{~V}$. The power was measured with a CEM DT-3348 RMS 1000A AC/DC watt clamp meter with an accuracy for alternative current (AC) power at $\pm 2.5 \%$. Note that the design of the unit is not compatible with the installation of any thermocouple, as the hot and cold interfaces of the bismuth telluride have a voltage. Estimations for the cold side temperature, cooled by forced ambient air convection, gives around $100^{\circ} \mathrm{C}$, while the hot side temperature is found to be below $300^{\circ} \mathrm{C}$. We used an infrared thermometer OMEGA OS423-LS and a thermocouple. We were able to measure the temperature near the cold side of the bismuth telluride and the exiting air temperature after the flowing between the hot side fins. We confirm in 2014 the measurements made in 2008: the 4-kW generator has an efficiency greater than twice the efficiency of a DC-operated thermoelectric generator operating between $300^{\circ} \mathrm{C}$ and $100^{\circ} \mathrm{C}$. No measurements on the two primary outlet circuits of this generator to the transformer of the DC-DC converter are possible.

The non-stationary analysis for the high frequency regime $(200 \mathrm{kHz})$ presented here confirms that operation with a DC-DC converter does have a frequency at which the impedance is very low, and that the impedance has an inductance-like character. It is this inductance which is used to extract the power from a thermoelectric generator with a low internal resistance, and the very low electrical impedance at a certain frequency may be the reason why the conversion efficiency is much greater than the DC-operated TEG. Such low electrical resistances are not possible with the commercially available thermoelectric modules, so the tested generator was designed with an internal electrical resistance of $3 \backslash \operatorname{sim} \mathrm{m} \Omega$. This type of TEG is difficult to build since all the thermoelectric elements must be soldered in one operation.

\section{CONCLUDING REMARKS}

Thermoelectric systems are driven by feedback processes between thermal and electrical currents. At low frequencies, the thermal constants dominate, but under specific conditions an electro-thermal coupling can occur at high frequency. The impedance spectroscopy reveals a non-resistive behaviour at high frequencies. DC-DC power conversion offers 
a large degree of freedom to the output impedance and the load matching. We have found on a commercial thermoelectric module from $\mathrm{Hi}-\mathrm{Z}$, a minimal impedance at a frequency around $6 \mathrm{kHz}$. Thermoelectric generators with a low internal resistance have an important reactive response. No impedance measurements at high frequencies have yet been made on such generators, but this is naturally the next step so that a mathematical model can be proposed and be validated experimentally. Only overall efficiency measurements on a thermoelectric generator designed with a very low internal resistance, operated in the hundred $\mathrm{kHz}$ range with a DC-DC converter, have been made with overall efficiencies at least twice greater than the DC-operated thermoelectric generators operating with the same temperature difference on the thermoelectric material.

\section{REFERENCES}

1. M. Nedelcu, M. Apostol, and J.G. Stockholm, IX Conference of the Polish Ceramic Society (Zakopane, September 2013). (in press)

2. M. Apostol and M. Nedelcu, J. Appl. Phys. 108, 023702 (2010).

3. Y. Apertet, H. Ouerdane, C. Goupil, and P. Lecoeur, Phys. Rev. E 88, 022137 (2013).

4. F.L. Curzon and B. Ahlborn, Am. J. Phys. 43, 22 (1975).

5. B. Andresen, Angew. Chem. Int. Ed. 50, 2690 (2011).

6. Y. Apertet, H. Ouerdane, O. Glavatskaya, C. Goupil, and P. Lecoeur, EPL 97, 28001 (2012).
7. Y. Apertet, H. Ouerdane, O. Glavatskaya, C. Goupil, and P Lecoeur, EPL 101, 68008 (2013).

8. Y. Apertet, H. Ouerdane, C. Goupil, and P. Lecoeur, Phys. Rev. E 85, 031116 (2012)

9. H. Ouerdane, Y. Apertet, C. Goupil, A. Abbout, and A. Michot, in Thermoelectric Nanomaterials, ed. by K. Koumoto, T. Mori (Springer Series in Materials Science, Berlin Heidelberg, 2013), pp. 323-351.

10. Y. Apertet, H. Ouerdane, C. Goupil, and P. Lecoeur, Phys. Rev. E 85, 041144 (2012).

11. P.E. Gray, The Dynamic Behavior of Thermoelectric Devices (M.I.T Press, Cambridge, 1960).

12. A.D. Downey, E. Timm, P.F.P. Poudeu, M.G. Kanatzidis, H. Shock, and T.P. Hogan, Mater. Res. Symp. Proc. 886, 425 (2005).

13. A.D. Downey and T.P. Hogan, Proceedings of the 24th International Conference on Thermoelectrics, Clemson, SC: 2005, pp. 79-82.

14. A.D. Downey, T.P. Hogan, and B. Cook, Rev. Sci. Inst. 78, 093904 (2007).

15. A.D. Downey, Advancing thermoelectric research with new measurement systems and thermoelectric modeling for AC electrical measurements, $\mathrm{PhD}$ dissertation (Michigan State University, 2006).

16. W. Park, M.T. Barako, A.M. Marconnet, M. Asheghi, and K.E. Goodson, Proceedings of 13th IEEE Intersociety Conference on Thermal and Thermomechanical Phenomena in Electronic Systems, ITherm 2012, pp. 107-112.

17. E. Hatzikkraniotis, K.T. Zorbas, I. Samaras, T. Kyratsi, and K.M. Paraskevopoulos, J. Electron. Mater. 39, 2112 (2010).

18. J. García-Cañadas and G. Min,J. Electron. Mater. 43, 2411 (2014).

19. M. Nedelcu, M. Apostol, and J.G. Stockholm, IX Conference of the Polish Ceramic Society (Zakopane, Poland, 2013).

20. B. Kim, L. Bun, C. Goupil, B. Dagues, and P. Maussion, Proceedings of the 2014 International Conference on Green Energy Sfax, Tunisia, 220, IEEE Xplore (2014).

21. J.M. Schroeder and G.P. Hirsch, Solid-state thermoelectric power converter, US patent: US 8101846 B1 (2012). 